data of the respondents through structured questionnaire. Data analysis was done using SPSS.

Results The respondents were mostly housewives on the age range of 20-34 years and finished Senior High School, officially married, but only half of them that living in Kupang officially married. Their husbands generally worked as nonprofessional and merchant. The sex behaviors of the pregnant women were categorized as not risky, but $47-78 \%$ having sex in the last week without condom. Only 10\% were referred to STI clinic for being checked up and receive consultation. Prevalence rates of infections on these pregnant women were: Chlamidiasis 5.9-6.6\%; Gonorrhea 0.6-0.7\%; Syphyilis 0$1.8 \%$; Trichomoniasis 0.6-9.5\%; Bacterial Vaginosis 18.865.1\%; Candidiasis 13-45,6\%; Herpes Simplex-2 IgM 0,739,2\%; Herpes Simplex-2 IgG 0-7,8\%; and HIV 0-1,8\%.

Conclusion The prevalence rates of RTIs-STIs in pregnant women in this study were high and as high as previous study in 1990s. This is urgent and need strategies to support more of the output of the program in all part of Indonesia as the rising of HIV prevalence in Indonesia.

Disclosure No significant relationships.

\section{P225 PRACTICAL COGNITIVE SCREENING FOR PATIENTS WITH HIV}

Gwen Levitt*. District Medical Group, Department of Psychiatry, Phoenix, USA

\subsection{6/sextrans-2019-sti.367}

Background In clinical practice it is imperative that patients with HIV, especially those with a concomitant mental illness, be screened for cognitive deficits. Not only is this important to document baseline levels of cognitive function, monitor for onset or progression of HIV-dementia, but to provide tools to improve treatment compliance for patients who demonstrate cognitive deficits. This study endeavored to determine what cognitive screening tools were most practical and effective to assess this population.

Methods HIV-positive participants were recruited from a psychiatric inpatient facility. Participants were administered five cognitive screening tools: Repeatable Battery for the Assessment of Neuropsychological Status (RBANS), Montreal Cognitive Assessment (MoCA), Mini-Mental Status Examination (MMSE), Trail Making Test- Parts A and B (TMT), and Wisconsin Card Sorting Test (WCST). The sample consisted of 21 participants with diagnoses including bipolar disorder (42\%), schizophrenia/schizoaffective disorder (25\%), depressive and anxiety disorder (17\%), psychotic disorder not otherwise specified (8\%), delusional disorder (4\%), and adjustment disorder (4\%). (Diagnostic and Statistical Manual of Mental Disorders- Fourth Edition was utilized.) Fifty-eight percent of the sample had co-occurring substance use disorders.

Results The mean age of participants was 42.08 years with 13.25 years of education. Ninety six percent of the sample were male. Fifty eight percent of the participants were Caucasian and 21\% were Hispanic and 21\% African American. Fifteen percent of the sample were newly diagnosed HIVpositive. Cognitive deficits were found on most of tools utilized. The RBANS demonstrated the most cognitive deficits consistent with the known literature in this population. The RBANS specifically revealed impairments in domains of delayed memory and attention. This, in turn, translates into problems retaining verbal and visual information.

Conclusion Development of cognitive assessment tools that can be utilized by non-psychologists to target this high-risk population is necessary as an important prognostic and treatment guide.

Disclosure No significant relationships.

\section{P226 SELF-ESTEEM, BODY IMAGE, AND SUBCULTURE IDENTIFICATION AMONG GAY, BISEXUAL, AND OTHER MEN WHO HAVE SEX WITH MEN}

${ }^{1}$ Kiffer Card*, ${ }^{2}$ Heather Armstrong, ${ }^{3}$ Shenyi Pan, ${ }^{3}$ Everett Blackwell, ${ }^{3}$ Marcus Greatheart, ${ }^{3}$ David Moore, ${ }^{3}$ Robert Hogg, ${ }^{4}$ Eric Roth, ${ }^{1}$ Nathan Lachowsky. ${ }^{1}$ University of Victoria, School of Public Health and Social Policy, Victoria, Canada; 'University of British Columbia, Vancouver, Canada; ${ }^{3}$ British Columbia Centre for Excellence in HIVIAIDS, Vancouver, Canada; ${ }^{4}$ University of Victoria, Victoria, Canada

\subsection{6/sextrans-2019-sti.368}

Background Previous research shows that low self-esteem may negatively affect self-efficacy, increase substance use, and underlie some patterns of risky sexual behaviour. This suggests low self-esteem may hinder the prevention of HIV and other sexually transmitted infections (STI). Therefore, we explored factors related to self-esteem among gay and bisexual men (gbMSM), including associations with risk behaviour.

Methods Between 2012-2015, we used respondent-driven sampling to recruit sexually-active HIV-positive and HIV-negative gbMSM, aged $\geq 16$ in Metro Vancouver. Participants completed visits every 6-month until 2018. Generalized estimating equations identified correlates of self-esteem (study $\alpha=.90$, range $=0-21$ ), including key measures of sexual behaviour, substance use, social embeddedness, body image, and sub-cultural identification.

Results Among 541 participants, 3,497 visits (Median: 7, Q - $^{-}$ $\left.\mathrm{Q}_{3}:[3-9]\right)$. In bivariable analyses, self-esteem scores did not differ by HIV-status ( $\mathrm{p}=0.59)$, and were not associated with seroadaptive behaviour ( $p>0.19$ across 7 strategies). After adjustment, higher self-esteem was associated with older age $(p<0.001)$, more social support $(p<0.001)$, larger social network size $(\mathrm{p}<0.003)$, lower emotional and social loneliness (each $\mathrm{p}<0.001)$, Asian $(\mathrm{p}=0.002)$ or Latin American $(p=0.001)$ identity (vs. White), higher self-report physical attractiveness $(p<0.005)$ and muscularity $(p<0.001)$, and subcultural identification as a Professional (versus not; $\mathrm{p}=0.04)$. While higher BMI was not associated with selfesteem $(p=0.94)$, identifying as a bear, cub, or otter (versus not; $\mathrm{p}=0.009)$ predicted lower self-esteem scores. Selfesteem was associated with several sexual (e.g., oral sex, masturbation, sex toy use) and substance use (i.e., binge drinking, cannabis, cocaine, methamphetamine) behaviours but these became non-significant after multivariable adjustment.

Conclusion Subculture identification, self-rated body-image, and social embeddedness are key to gbMSM's self-esteem. Given these factors overshadowed self-esteem's association with sexual and substance use behaviours, holistic HIV and STI interventions should leverage socially-supportive, community-based, and inclusive messaging to address potentially negative effects of low self-esteem on gbMSM's sexual health. Disclosure No significant relationships. 